\title{
Special issue on 'Analytical and computational methods in probability theory and its applications conference'
}

\author{
Dmitry Korshunov ${ }^{1}$ - Alexander Veretennikov ${ }^{2}$ \\ Published online: 7 March 2020 \\ (c) Springer Science+Business Media, LLC, part of Springer Nature 2020
}

This special issue contains a selection of papers written by the participants of the international conference Analytical and Computational Methods in Probability Theory and its Applications-ACMPT-2017 which was held on October 23-27, 2017, at Lomonosov Moscow State University and RUDN University, Russia. The conference was organized under the auspices of the Moscow Mathematical Society.

The conference was dedicated to the 90th birth anniversary of Alexander Solov'ev (Solovyev), an outstanding Russian probabilist and specialist in queueing theory (6.09.1927-6.04.2001). A nearly complete list of publications of A. Solov'ev can be found in [1]. Almost 200 talks were given, they presented recent developments in analytical, computational and asymptotic methods in probability theory and its applications with special emphasis on the fields of primary interest of Solov'ev, which include but are not restricted to queueing systems, reliability and risks, limit theorems, etc. The conference was attended by many experts in these areas, in particular, by many colleagues and former students of A. D. Solov'ev.

The special issue includes two papers. In the first of them, Stability conditions for a multiserver queueing system with a regenerative input flow and simultaneous service of a customer by a random number of servers, Larisa Afanaseva and Svetlana Grishunina consider a multiserver queueing system where each customer requires a random number of servers simultaneously. The input flow is assumed regenerative, while service times are either exponential, phase type or hyper-exponential. Following the synchronization method, the authors establish stability criteria for such systems. The main contribution of the paper is an extension of the stability criteria to the case of regenerative input flow. The class of regenerative flows is really broad and particularly includes Markov arrival processes, doubly stochastic Poisson processes with a regenerative process as intensity, and Markov modulated processes, among others. It is shown in the paper that the only characteristic of the input flow that plays a role for stability is its intensity, while the structure of the input flow does not play

\footnotetext{
$\triangle$ Dmitry Korshunov

d.korshunov@lancaster.ac.uk

1 Lancaster University, Lancaster, UK

2 University of Leeds, Leeds, UK
} 
any role. Concerning the service time distribution, the situation is different and stability depends on that distribution. The authors provide counter examples that demonstrate that the stability condition cannot be expressed in terms of the service time mean.

The second paper, On mean-field GI/GI/1 queueing model: existence and uniqueness by Alexander Veretennikov, is related to a mean-field extension of the single-server queueing system. Under some conditions on arrival and service intensities, the corresponding process is constructed as a Markov solution to a martingale problem. The author considers the case where intensities may depend on the state of the process as well as on the marginal distribution of the process. The uniqueness in distribution is established next, under a slightly different set of assumptions on intensities. The topic of this paper is related to the so-called McKean-Vlasov models in stochastic analysis.

\section{Reference}

1. Gnedenko, D.B., Demidov, S.S., Zubkov, A.M., Kashtanov, V.A.: A. D. Soloviev. In: Rykov, V., Singpurwalla, N., Zubkov, A. (eds.) Analytical and Computational Methods in Probability Theory (ACMPT 2017). Lecture Notes in Computer Science, vol. 10684. Springer, Cham (2017)

Publisher's Note Springer Nature remains neutral with regard to jurisdictional claims in published maps and institutional affiliations. 